Bio - grafía. Escritos sobre la Biología y su Enseñanza. ISSN 2027-1034

Edición Extraordinaria. p.p. 1157 - 1163

Memorias del IX Encuentro Nacional de Experiencias en Enseñanza de la Biología y la Educación Ambiental. IV Congreso Nacional de Investigación en Enseñanza de la Biología.

\title{
INTEGRACIÓN DE TIC Y ABP EN ENSEÑANZA DE LA QUÍMICA ORGÁNICA PARA ESTUDIANTES DE LICENCIATURA EN BIOLOGÍA
}

\section{ICT AND PBL INTEGRATION INTO ORGANIC CHEMISTRY TEACHING FOR BIOLOGY TEACHER STUDENTS}

\section{Nubia Farid Barrera Cobos ${ }^{1}$}

\section{RESUMEN}

Es imprescindible hoy en día en Colombia que un docente de Biología tenga conocimientos en Química dado que las condiciones laborales exigen a los profesionales encargarse de los cursos tanto de biología como de química razón por la cual una buena adquisición de conceptos y de nociones de química le permitirá competir en el mundo laboral. El presente trabajo muestra la integración de tecnologías de la información y la comunicación (TICs) con la metodología de aprendizaje basado en problemas (ABP) para estudiantes de Licenciatura en Biología, el proceso involucra sesiones magistrales en donde los estudiantes deben consultar las funciones químicas orgánicas, participar dentro de las discusiones que se generan en clase y la creación de un blog en grupo para la descripción de una función química orgánica en particular siguiendo las instrucciones dadas para la presentación. El objetivo del trabajo era utilizar otras metodologías de aprendizaje para que los estudiantes observaran la relación entre la teoría y la práctica, y de estas con las moléculas que hacen parte de los sistemas biológicos y utilizaran las TICs para fomentar las competencias genéricas y específicas del curso. En conclusión, los estudiantes lograron apropiar el conocimiento relacionando los contenidos con moléculas biológicas y se apropiaron de otras formas de difusión de conocimiento utilizando TIC.

\section{PALABRAS CLAVE}

TIC's, Tecnolog'ias de la información y la comunicación, ABP, Aprendizaje basado en problemas, química orgánica, sistemas biológicos, moléculas biológicas.

\section{ABSTRACT}

It is imperative today in Colombia that a Biology teacher has knowledge in Chemistry. Working conditions require professionals to take care of both biology and chemistry

\footnotetext{
${ }^{1}$ Candidato a Doctor en Biotecnología, Magister en Bioquími ca, Licenciada en Química. UDFJ. Docente de Química Orgánica y Bioquímica del Proyecto Curricular licenciatura en Biología de la Universidad Distrital Francisco José de Caldas. Docente de la Escuela Colombiana de Rehabilitación. Email: nubiafarid50@yahoo.com
} 


\section{Bio - grafía. Escritos sobre la Biología y su Enseñanza. ISSN 2027-1034}

\section{Edición Extraordinaria. p.p. 1157 - 1163}

Memorias del IX Encuentro Nacional de Experiencias en Enseñanza de la Biología y la

Educación Ambiental. IV Congreso Nacional de Investigación en Enseñanza de la

Biología.

courses. A good conceptual base in chemistry will allow the biology teacher better access to the labor market. The present work shows the integration of information and communication technologies (ICT) with the problem-based learning methodology (PBL) applied to undergraduate students in Biology. The process involves keynote sessions where students should consult organic chemical functions, participate in the discussions that are generated in class and the creation of a group blog for the description of a particular organic chemical function, following the given instructions. The aim of the work was to use other learning methodologies so that the students observed the relationship between theory and practice, and those with the molecules that are part of the biological systems and use the ICTs to promote the generic and specific competences of the course. The results showed that students were able to appropriate knowledge by relating the contents to biological molecules and appropriated other forms of knowledge diffusion using ICTs.

\section{KEYWORDS}

ICT, Information and communication technologies, PBL, problem-based learning methodology, Organic chemistry, Biological systems, biological molecules.

\section{INTRODUCCIÓN}

El proceso educativo presenta hoy en día varias posibilidades para que se puedan realizar diversos metodologías que le permitan al estudiante comprender los diferentes conceptos de una determinada asignatura; es así como la química orgánica se presenta como una asignatura de memoria en donde el estudiante debe aprender una serie de funciones químicas orgánicas, la nomenclatura, la síntesis y las reacciones que producen cada una de esas funciones químicas orgánicas. Para el estudiante de Biología se hace bastante tedioso el aprendizaje de las funciones químicas y el uso de la memoria y los procesos de análisis y deducción son bastante deficientes dentro del proceso; lo cual no significa que los estudiantes puedan llegar a realizar eficientemente los diferentes procedimientos que se les proponen. En este aspecto el uso de diferentes metodologías de aprendizaje en donde los estudiantes utilizan plataformas para la realización de actividades de nomenclatura o la creación de Blogs en donde relacionen las funciones químicas orgánicas con compuestos biológicos de importancia cobran interés para el estudiante cuando realizan estas interacciones y se presenta un aprendizaje significativo.

\section{METODOLOGÍA ABP}

El ABP o PBL es una metodología de aprendizaje constructivista en donde el estudiante es el centro de la actividad educativa (Barrows, 1980), en este escenario se plantean problemas como puntos iniciales para la adquisición, fijación e integración de nuevo conocimiento; en este caso el problema consistió en la creación de un blog de química orgánica en donde se revisarán aspectos importantes dentro de una función química 
Bio - grafía. Escritos sobre la Biología y su Enseñanza. ISSN 2027-1034

Edición Extraordinaria. p.p. 1157 - 1163

Memorias del IX Encuentro Nacional de Experiencias en Enseñanza de la Biología y la

Educación Ambiental. IV Congreso Nacional de Investigación en Enseñanza de la

Biología.

orgánica en particular, y en donde se describieran las reacciones de síntesis y las reacciones que producen las funciones químicas e integraran la práctica de laboratorio que se realizó soportando con el análisis de resultados la reacción obtenida.

Además del aprendizaje de los conceptos, muy importante para el docente que los estudiantes desarrollen habilidades como responsabilidad, capacidad de comunicación, capacidad de análisis, creatividad, emprendimiento, aprendizaje y trabajo en equipo (Duc et al., 2001). Las competencias específicas que los estudiantes deben cumplir son la aplicación de los conceptos adquiridos y la relación con las moléculas biológicas.

Esta metodología permite que los estudiantes trabajen de forma individual y grupal, utilizando procedimientos secuenciales que les permitan resolver el problema planteado así como aprender a autoevaluarse y evaluar a sus compañeros y docente (Jorba J \& Sanmartí N, 1996)

Las características principales de esta metodología son: aprendizaje centrado en el estudiante, creación de grupos pequeños para fomentar el aprendizaje, los docentes son facilitadores, autodirección del aprendizaje. (Molina et al, 2003).

El uso del ABP permite que los estudiantes generen aprendizaje significativo (Ausubel, 1983) y que los prepare para el campo laboral y que les permita tener las herramientas para alcanzar las metas profesionales que se propongan. Existen diferentes tipologías de ABP que se pueden utilizar para que los estudiantes puedan aprender:

a. Basado en un tema

b. Basado en una pregunta

c. Basado en un problema

Uso de TIC's

La universidad no es ajena al uso de las tecnologías de la comunicación e información (Moreno N., 2011), teniendo en cuenta que hoy en día los jóvenes utilizan diversas maneras de comunicarse y de relacionarse haciendo uso de la tecnología; el uso de estas metodologías permite generar diversas actividades y modalidades de enseñanza aprendizaje que les permite plantear nuevas alternativas y renovar ideas y paradigmas. (Arnau et al, 2002).

La combinación de la metodología ABP junto con las estrategias de enseñanza aprendizaje le puede permitir al estudiante generar procesos metacognitivos y ampliar su conocimiento respecto a un tema determinado (Klimenko O. \& Alvares J., 1996) 


\section{Bio - grafía. Escritos sobre la Biología y su Enseñanza. ISSN 2027-1034}

\section{Edición Extraordinaria. p.p. 1157 - 1163}

Memorias del IX Encuentro Nacional de Experiencias en Enseñanza de la Biología y la

Educación Ambiental. IV Congreso Nacional de Investigación en Enseñanza de la

Biología.

\section{METODOLOGÍA}

El presente trabajo se realizó con estudiantes de segundo semestre de la carrera de Licenciatura en Biología de la Universidad Distrital Francisco José de Caldas. La asignatura de química orgánica involucra la descripción de las funciones químicas orgánicas con los temas relacionados entre ellas y la adquisición de los conocimientos básicos para que el estudiante logré desarrollar procesos de análisis y de síntesis de información. A su vez, el espacio académico propende por un aprendizaje activo de los estudiantes en donde se dirigen las actividades para que los educandos participen activamente de las sesiones, para lo cual deben realizar lecturas específicas de temas en libros de texto antes de cada sesión de clase, con lo cual preparan la sesión presencial y tienen bases para realizar preguntas y responderlas allí. Igualmente se realizan trabajos en el aula virtual del espacio académico con el fin de que los estudiantes puedan integrar el conocimiento y además puedan cumplir las competencias del espacio académico.

Las participaciones tanto en el aula virtual como en las sesiones presenciales permiten a los estudiantes alcanzar las competencias de forma rápida que aquellos que no lo hacen, permitiendo obtener mejores resultados a lo largo del curso y por lo tanto lo puedan aprobar. Para el desarrollo del proceso educativo se realizaron sesiones magistrales de química orgánica, en donde la docente explicó las funciones químicas orgánicas, las relacionó con moléculas biológicas, identificó aquellas moléculas de relevancia para el estudiante, generó cuadros de proceso de síntesis de la función química y reacciones que producen las funciones químicas, al final de la sesión se dejó tarea y consulta para discusión en la siguiente sesión. Las sesiones permitían la discusión de los temas de consulta y la retroalimentación por parte del docente; a su vez se realizaron evaluaciones con el fin de corroborar el aprendizaje de los estudiantes.

La continuación y profundización de cada tema se realizó mediante la metodología de aprendizaje basado en problemas teniendo como base un tema, que para el caso del espacio académico consistió en una función química orgánica; los estudiantes organizados en grupos seleccionaron de forma libre el tema de interés y utilizaron la estrategia de crear un blog del tema en el cual se incluyeron aspectos relevantes para un licenciado en Biología como la nomenclatura, usos comunes, moléculas de importancia biológica y la forma de identificación en el laboratorio de estos compuestos químicos, teniendo como base el desarrollo del laboratorio por el grupo de estudiantes, de lo cual se realizó un informe de laboratorio y se tomaron fotografía<s que fueron colocadas dentro del blog; así mismo los estudiantes recibieron la sesión magistral sobre el tema de interés y explicaron en el blog mediante reacciones químicas cada una de las que se realizaron en la práctica de laboratorio, la creación del blog, el diseño y la organización de los temas estuvo a cargo de los estudiantes y la forma creativa como decidieron hacerlo.

Para la realización del blog los estudiantes debieron recurrir además a textos de estudio, complementar la información obtenida mediante consultas del tema y de las reacciones de laboratorio y del correcto análisis de los mismos, ya que el informe de laboratorio correspondiente se entregó antes de la realización del blog al docente para su evaluación.

Para valorar este proceso se aplicaron evaluaciones que permitieron ver el nivel de avance en el aprendizaje, utilizando para ello mapas de reacciones químicas orgánicas, 


\section{Bio - grafía. Escritos sobre la Biología y su Enseñanza. ISSN 2027-1034}

\section{Edición Extraordinaria. p.p. 1157 - 1163}

Memorias del IX Encuentro Nacional de Experiencias en Enseñanza de la Biología y la

Educación Ambiental. IV Congreso Nacional de Investigación en Enseñanza de la Biología.

cuya finalidad es determinar el grado de análisis que tienen los estudiantes de Licenciatura en Biología.

Para el caso de la integración de las TIC's con ABP se generaron grupos de trabajo de máximo 4 estudiantes y se eligió al azar una función química orgánica por grupo con el fin de que los grupos de estudiantes realizaran una consulta del tema y crearan un blog en la web; las funciones químicas orgánicas que se seleccionaron fueron aquellas que pueden relacionarse con moléculas biológicas: alcanos, alquenos, compuestos aromáticos, alcoholes, fenoles, éteres, compuestos carbonílicos, ácidos carboxílicos, vitaminas, aminoácidos.

Paralelo al proceso los estudiantes asistieron a sesiones de laboratorio en donde realizaron prácticas relacionadas con la identificación de la función química orgánica. Terminada la sesión de laboratorio, los estudiantes presentaron un informe de laboratorio de forma grupal en donde deberían estar consignadas las reacciones correspondientes con su análisis tomado de textos de estudio, se permitió tomar fotos de los experimentos realizados para una mejor comparación de los resultados.

Para finalizar el curso, los estudiantes diseñaron un blog en donde debieron consignar la función química orgánica, las moléculas de importancia, las reacciones que realizaron en el laboratorio con su fundamentación, todo muy bien referenciado

\section{RESULTADOS Y DISCUSIÓN}

Como resultado del proceso, los estudiantes mediante las prácticas académicas lograron afianzar sus conocimientos en química orgánica y realizaron informes que mejoraron con el transcurso del curso, ya que al inicio del mismo los estudiantes no entendían muy bien la estructuración del proceso de análisis de los resultados obtenidos, la consulta ni la referenciación; mediante los procesos de tutorías y de seguimiento que se impartió en cada una de las sesiones, se logró un gran avance en cuanto a la forma de presentar los informes y de realizarlos; así los estudiantes comprendieron el proceso de realización de prácticas y lo relacionaron con la teoría mediante la consulta de preguntas clave que se realizaron.

Así mismo, las prácticas fueron relacionadas con la creación de un blog que se generó por grupos de estudiantes, éstos tuvieron diferentes formatos cumpliendo con las competencias de responsabilidad, creatividad, y aprendizaje y trabajo en equipo; incluso muchos grupos se atrevieron a realizar actividades lúdicas con el fin de medir lo aprendido dentro del blog. Las citas y referencias bibliográficas fueron oportunas en cada caso y se valieron de gráficas y de documentos citados para escribir las reacciones vistas en clase.

En la evaluación del trabajo ABP se consideran aspectos como: presentación, creatividad, uso de citas bibliográficas, lenguaje utilizado, tipo y calidad de información presentada por cada grupo, el diseño original de cada grupo permitió ver el alcance y la relevancia que 
Bio - grafía. Escritos sobre la Biología y su Enseñanza. ISSN 2027-1034

Edición Extraordinaria. p.p. 1157 - 1163

Memorias del IX Encuentro Nacional de Experiencias en Enseñanza de la Biología y la

Educación Ambiental. IV Congreso Nacional de Investigación en Enseñanza de la

Biología.

los estudiantes le imprimen a cada tema, además de darles libertad en la diagramación y en la forma de presentación, lo cual también permite que el conocimiento sea más cercano a la comunidad.

Estos procesos de enseñanza - aprendizaje comprueban que los estudiantes pueden realizar diferentes procesos de apropiación del conocimiento y de transmisión del mismo mediante la incorporación de diversas técnicas como las TIC y que pueden generar contenidos que pueden divulgarse mediante el uso de las mismas a la vez que involucran metodologías de aprendizaje basado en problemas como método de búsqueda y de aprendizaje significativo, colaborativo y de resolución de problemas utilizando procesos creativos.

La mayoría de los estudiantes han expresado que han aprendido a expresar conceptos y conocimientos nuevos a partir de la metodología y que les ha parecido interesante la combinación de las TIC's en el proceso de enseñanza - aprendizaje, igualmente que han logrado las competencias descritas para el curso de Química orgánica.

\section{CONCLUSIONES}

Es innegable el alcance que hoy en día se tienen de las tecnologías de la información y de los diferentes procesos como los estudiantes pueden acceder al conocimiento, por lo cual la integración de procesos en los cuales los alumnos puedan integrar sus conocimientos, su creatividad y su compromiso con el aprendizaje permiten que se genere un aprendizaje significativo que conduce a un buen cumplimiento de las competencias del espacio académico además de que los motiva hacia el uso de herramientas que los entusiasma por la obtención de nuevo conocimiento y la forma como pueden transmitirlo, haciendo además uso de sus facultades como docentes y de la facilidad en el uso de otras herramientas que pueden complementar el trabajo en equipo. Además este tipo de actividades fomenta la comunicación entre los estudiantes y entre ellos y la docente.

\section{BIBLIOGRAFÍA}

Ausubel, D.P. et al. (1983). Psicología Educativa. México: Ed. Trillas

Arnau, J.; Bono, R.; García, M. (2002). «Integración de nuevas tecnologías en la docencia universitaria». 20 Congreso Internacional de Docencia Universitaria e Innovación. Tarragona.

Barrows, H. S.; Tamblyn, R. M. (1980). Problem Based Learning. Nueva York: Springer

Duch, B. J. \& Allen D. E. (2001). Why Problem-Based Learning? A Case Study of Institutional Change in Undergraduate Education. Virginia: Stylus Publishing. 3-11. 
Bio - grafía. Escritos sobre la Biología y su Enseñanza. ISSN 2027-1034

Edición Extraordinaria. p.p. 1157 - 1163

Memorias del IX Encuentro Nacional de Experiencias en Enseñanza de la Biología y la Educación Ambiental. IV Congreso Nacional de Investigación en Enseñanza de la Biología.

Jorba, J. \& Sanmartí, N. (1996). Enseñar, Aprender y Evaluar: Un Proceso de Regulación Continua. Propuestas Didácticas para el Área de Ciencias de la Naturaleza y Matemáticas. MEC: Madrid.

Klimenko, O. \& Alvares, J. L. (2009). Aprender cómo aprendo: la enseñanza de estrategias metacognitivas. En: Investigación pedagógica. Vol. 12. No 198.11-28 p.

Molina, J.A., García, A., Pedraz, A. y Mª Victoria (2003) Aprendizaje basado en problemas: una alternativa al método tradicional. Revista de la Red Estatal de Docencia Universitaria, 3: (2).

Moreno, N. M. (2011). Las Tics como herramientas para el desarrollo del aprendizaje autónomo del español como segunda lengua en las A.T.A.L. en: XII Congreso internacional de Teoría de la educación. Universidad de Barcelona. 